\title{
Hypofractionated external beam radiation therapy in combination with HDR boost for localized prostate cancer: patient reported quality of life outcomes
}

\author{
Tiffany M. Morgan, MD', Robert H. Press, MD!, Patrick K. Cutrell, MS!, Chao Zhang, PhD², Zhengjia Chen, PhD², \\ Sara Rahnema, MS, DABR', Jaymin Jhaveri, MD!, Martin Sanda, MD³, John Pattaras, MD³, Pretesh Patel, MD!, \\ Ashesh B. Jani, MD!, Peter J. Rossi, MD' \\ 'Department of Radiation Oncology, The Winship Cancer Institute, Emory University, Atlanta, ${ }^{2}$ Department of Biostatistics and Bioinformatics, \\ The Winship Cancer Institute, Emory University. Atlanta, ${ }^{3}$ Department of Urology. The Winship Cancer Institute, Emory University. Atlanta, USA
}

\begin{abstract}
Purpose: There is limited data to support the use of hypofractionated external beam radiation (HypoF) in combination with high-dose-rate brachytherapy (HDR). We report our quality of life (QOL) outcomes when treating intermediate and high-risk prostate cancer patients with external beam radiation (EBRT) plus HDR.

Material and methods: The charts of 54 patients with localized adenocarcinoma of the prostate treated with standard fractionation (SF) or HypoF EBRT plus HDR boost at a single institution between 2012 and 2015 were reviewed. All patients completed the American Urological Association Symptom Score (AUASS) and Expanded Prostate Index for Prostate Cancer - Clinical Practice (EPIC-CP) quality of life assessments prior to treatment and completed at least one follow-up survey. Linear mixed models were performed to test for significant changes and differences in each outcome over time.
\end{abstract}

Results: There was no significant difference in AUA score $(p=0.98)$, incontinence (urge) and urinary irritation/ obstruction scores ( $p=0.81$ and $p=0.62$, respectively), and bowel QOL ( $p=0.97$ ) between the two dosing groups over time or at any discrete time point. For both groups, AUA scores peaked at 0-2 months before improving. Likewise, sexual function, vitality score, and QOL scores were also not significantly different between the dose groups over time $(p=0.59, p=0.37$, and $p=0.71$, respectively). All QOL categories, except sexual function, trended toward baseline with increasing time from intervention.

Conclusions: Our study suggests HypoF EBRT can be delivered in combination with HDR for patients with ntermediate-risk and high-risk adenocarcinoma of the prostate without increasing toxicity compared to SF with an HDR boost.

Key words: boost, brachytherapy, quality of life, HDR, prostate cancer.

\section{Purpose}

Radiation dose escalation has long been associated with improved biochemical relapse-free survival in prostate cancer. Initially, dose escalation was achieved using external beam radiation therapy (EBRT) alone [1,2,3,4,5]. The advent of intensity-modulated radiation therapy (IMRT) and image-guided radiation therapy (IGRT) allowed for more conformal treatments and decreased toxicity $[6,7,8]$. More recently, brachytherapy has been used as a means of dose escalation, given the rapid dose fall-off associated with the technique and improved sparing of normal tissue. Three randomized trials including intermediate and high-risk prostate cancer patients have shown improvement in biochemical recurrence-free survival when escalation is achieved with a brachytherapy boost added to EBRT $[9,10,11]$. In one of the trials, the control arm consisted of modern external beam doses, leading to EBRT plus a brachytherapy boost to be considered a standard of care for intermediate and high-risk patients able to tolerate a procedure [11].

Given the importance of cost effective treatment strategies in combination with the low alpha/beta ratio associated with prostate cancer, hypofractionated treatment
Address for correspondence: Tiffany M. Morgan, MD, Department of Radiation Oncology, The Winship Cancer Institute of Emory University, 1365 Clifton Road NE, Room C-5092, Atlanta, GA 30322, USA, phone: +1 404 778 3473, fax: +1 404778 4139, $\bowtie$ e-mail: tiffany.morgan2@emory.edu
Received: 19.04 .2018

Accepted: 18.06 .2018

Published: 30.06 .2018 
schedules have been investigated [12]. Four randomized trials have shown no difference in treatment efficacy when a hypofractionated EBRT treatment is delivered rather than a conventionally fractionated EBRT treatment $[13,14,15,16]$. This has become the new standard of care at many institutions, which improves resource utilization and is more convenient for patients. However, there is limited evidence on combining hypofractionated EBRT with a brachytherapy boost [17]. At our institution, EBRT plus a brachytherapy boost is delivered to unfavorable intermediate and high-risk prostate cancer patients whenever feasible. Since 2013, whenever possible, the external beam portion of the treatment has been delivered via a hypofractionated regimen, as discussed in the American College of Radiology (ACR) Appropriateness Criteria [18]. We report our quality of life (QoL) outcomes between patients receiving standard vs. hypofractionated EBRT and a high-dose-rate brachytherapy (HDR) boost.

\section{Material and methods}

After institutional review board approval, the charts of 202 patients with biopsy proven localized adenocarcinoma of the prostate who received brachytherapy at our institution between 2012 and 2015 were retrospectively reviewed. Patients who were treated with standard or hypofractionated EBRT plus HDR boost were included in the analysis. Patients were excluded if their prostate cancer was previously treated. Patient reported outcomes (PRO) were collected at baseline and at regular intervals after treatment using the American Urological Association Symptom Score (AUASS) and Expanded Prostate Index for Prostate Cancer - Clinical Practice (EPIC-CP). The American Urological Association developed the AUASS to determine how bothersome men's urinary symptoms are. Each urinary symptom is given a score from $0-5$. A score of 0 means the symptoms do not occur at all, while a score of 3 means men experience the symptoms about half the time. A score of 5 means the symptoms occur almost always. The AUASS consists of 7 urinary specific questions and is scored out of a total of 35 points. EPIC-CP is divided into subdomains, including urinary incontinence (incon), urinary irritability and obstructive symptoms (irr/obs), bowel QoL, sexual function, and vitality. Each subdomain consists of 3 questions and is scored out of a total of 12 points, while the overall EPIC-CP quality of life score is out of a total of 60 points. All patients completed the AUASS and EPIC-CP quality of life assessments prior to treatment and completed at least one follow-up survey. This left 54 patients for analysis.

\section{External beam radiation therapy}

For all patients, EBRT was delivered prior to brachytherapy. The CTV standardly consisted of the prostate and seminal vesicles. However, pelvic lymph nodes were included in the CTV for 6 patients with high-risk disease. A 0.3-1 cm margin was added to the CTV to create the PTV. All patients treated with standard fractionation EBRT received $45 \mathrm{~Gy}$ in 25 fractions $(n=38)$. Hypofractionation regimens included $37.5 \mathrm{~Gy}$ in 15 fractions $(n=14)$, 42.5 Gy in 17 fractions $(n=1)$, and 40 Gy in 16 fractions $(n=1)$. Assuming an alpha/beta of 1.5 for prostate cancer, the biologically equivalent dose (BED) was calculated for each EBRT regimen and summed with the BED for each HDR regimen for the total BED (Table 1). All patients were treated with either intensity-modulated radiation therapy (IMRT) using a fixed gantry technique, or volumetric-modulated arc radiotherapy (VMAT) under daily image guidance.

\section{High-dose-rate brachytherapy}

HDR brachytherapy was carried out in an operating room. First, biplanar ultrasonography was performed in transverse and sagittal dimensions to identify the prostate and seminal vesicles, along with normal structures at risk, including the urethra, bladder, and rectum. A transperineal interstitial brachytherapy template was used to place 5 or 6-French HDR catheters into the prostate, under ultrasound guidance, at the appropriate depth circumferentially around the peripheral capsule of the prostate. Care was taken to avoid the urethra. After the catheters were placed and the template secured, the patient was transferred to the radiation oncology department where CT simulation was performed to verify brachytherapy catheter depth and for treatment planning. Treatment was delivered to the target volume using a remote after loaded Ir-192 source. Brachytherapy doses included 9.5 Gy $\times 2$ fractions $(n=38), 10$ Gy $\times 2$ fractions $(n=10)$, 10.5 Gy $\times 2$ fractions $(n=3)$, or 15 Gy $\times 1$ fraction $(n=4)$. HDR dosimetry details are listed in Table 2.

\section{Androgen deprivation therapy}

Androgen deprivation therapy (ADT) was standardly delivered via an LHRH agonist with or without casodex to patients with unfavorable intermediate- and highrisk disease when feasible. ADT was typically given for

Table 1. Cumulative $B E D_{1.5}$ (Gy) for each combination of EBRT and HDR

\begin{tabular}{lccccc} 
EBRT dose (total dose in Gy/number of fractions) & $45 / 25$ & $37.5 / 15$ & $42.5 / 17$ & $40 / 16$ \\
\hline HDR dose (dose per fraction in Gy $\times$ number of fractions) & & & & \\
\hline $9.5 \times 2$ & 238.33 & 239.33 & 252.55 & 246 & 260 \\
\hline $10 \times 2$ & 252.33 & 253.33 & 266.66 & - \\
\hline $10.5 \times 2$ & 267 & - & - & 278.33 & 27.67
\end{tabular}

EBRT - external beam radiation, $B E D_{1.5}$ - biologically equivalent dose at an alpha/beta of 1.5, HDR - high-dose-rate brachytherapy 
Table 2. High-dose-rate dosimetry details

\begin{tabular}{|c|c|c|c|}
\hline Variable & Standard fractionation $(n=38)$ & Hypofractionation $(n=16)$ & $p$-value \\
\hline Mean prostate volume (cc) & 44.2 (range, 21.58-84.1) & 41.9 (range, 28.16-72.78) & 0.583 \\
\hline HDR dose & & & 0.775 \\
\hline 9.5 Gy $\times 2$ fractions & $26(68.4 \%)$ & $11(68.8 \%)$ & \\
\hline 10 Gy $\times 2$ fractions & $6(15.8 \%)$ & $4(25.0 \%)$ & \\
\hline 10.5 Gy $\times 2$ fractions & $3(7.9 \%)$ & 0 & \\
\hline 15 Gy $\times 1$ fraction & $3(7.9 \%)$ & $1(6.3 \%)$ & \\
\hline Median $\mathrm{D}_{90}(\%)$ & $104.85(92.4-111.9)$ & $105.01(100.31-112.30)$ & \\
\hline Median $\mathrm{V}_{100}(\%)$ & $94.3(83.3-105.04)$ & $95.21(90.23-99.19)$ & \\
\hline Median $V_{200}(\%)$ & $13.0(6.15-19.3)$ & $12.22(10.57-17.12)$ & \\
\hline Mean rectal $\mathrm{V}_{75 \%}(\mathrm{cc})$ & $0.058(0.00-0.39)$ & $0.044(0.00-0.19)$ & \\
\hline Mean bladder $\mathrm{V}_{75 \%}(\mathrm{cc})$ & $0.070(0.00-0.63)$ & $0.03(0.00-0.38)$ & \\
\hline Mean urethral $\mathrm{V}_{125 \%}(\mathrm{cc})$ & $0.006(0.00-0.05)$ & $0.008(0.00-0.07)$ & \\
\hline
\end{tabular}

6 months for those with unfavorable intermediate disease, and for 6-24 months for those with high-risk disease. Treatment details are listed in Table 3.

\section{Statistical analysis}

The groups analyzed included hypofractionated EBRT plus HDR boost vs. standard fractionation EBRT plus HDR boost. The univariate association of each covariate with the two groups, hypofractionation, and standard fractionation was assessed using the $\chi^{2}$ test for categorical covariates and $t$ test for numerical covariates. Outcomes include AUA score and EPIC-CP overall prostate cancer QoL score and subdomain scores - urinary incontinence (incon), irr/obs, bowel QoL, sexual function, and vitality. All outcome variables were treated as continuous variables. For descriptive statistics of each outcome, time points were set up as baseline, 0-2 months, 3-6 months, 7-12 months, 13-18 months, $19-24$ months, $25-30$ months, and $>30$ months. The mean and standard deviation of the outcomes of the different treatment groups at different measurement time points were calculated. Linear mixed models were performed to test whether there was any significant change over time for each outcome, and to detect whether there was any significant difference in each outcome among different treatments. The significance level was set at 0.05 . SAS 9.4 was used for data analyses and management.

\section{Results}

\section{Patient characteristics}

Fifty-four patients with localized prostate adenocarcinoma were included in the analysis. Median age at treatment was 68 years (range, 55-82 years). Median follow-up for the standard fractionation and hypofractionation cohorts was 24 months (range, 2-51 months) and 19 months (range, 2-40 months), respectively. A majority of patients had clinical T1c disease $(n=35)$, a Gleason score of 7 $(n=35)$, and a PSA $<10(n=39)$. Thirty-one patients had NCCN intermediate-risk disease and 23 had high-risk disease. Baseline characteristics were well-balanced between the 2 groups (Table 3 ).

\section{Hypofractionated vs. standard fraction external beam radiation therapy}

\section{Urinary function}

There was no significant difference in AUA score between the two dosing groups over time $(p=0.98)$, nor were there any significant differences at any discrete time point (Figure 1). Likewise, incon and irr/obs scores were not significantly different between the two groups over time $(p=0.81$ and $p=0.62$, respectively) (Figure 1). Incon and irr/obs scores did not differ between the two groups at any time point. For both groups, AUA and irr/obs scores peaked at 0-2 months before improving. Incontinence scores peaked at 3-6 months in the hypofractionation group, and at 19-24 months in the standard fractionation group. No patients in either group required temporary catheterization or developed a urethral stricture after treatment.

\section{Bowel quality of life}

Bowel toxicity peaked at 7-12 months in the hypofractionation group and at $0-2$ months in the standard fractionation group. However, bowel QoL was not significantly different between either group over time $(p=0.97)$, or at any discrete time point (Figure 2).

\section{Sexual function}

While sexual dysfunction was not significantly different between the dose groups over time $(p=0.59)$ (Figure 2), 
Table 3. Patient, disease, and treatment characteristics

\begin{tabular}{|c|c|c|c|}
\hline Variable & Standard fractionation $(n=38)$ & Hypofractionation $(n=16)$ & $p$-value \\
\hline Median age (years) (range) & $68(55-82)$ & $67(60-77)$ & 0.776 \\
\hline Clinical T stage & & & 0.901 \\
\hline T1c & $25(65.8 \%)$ & $10(62.5 \%)$ & \\
\hline $\mathrm{T} 2 \mathrm{a}$ & $6(15.8 \%)$ & $3(18.8 \%)$ & \\
\hline$T 2 b$ & $1(2.6 \%)$ & $1(6.3 \%)$ & \\
\hline $\mathrm{T} 2 \mathrm{C}$ & $2(5.3 \%)$ & $1(6.3 \%)$ & \\
\hline T3a & $2(5.3 \%)$ & $1(6.3 \%)$ & \\
\hline $\mathrm{T} 3 \mathrm{~b}$ & $1(2.6 \%)$ & 0 & \\
\hline PSA & & & 0.583 \\
\hline$<10$ & $26(68.4 \%)$ & $13(81.3 \%)$ & \\
\hline $10-20$ & $10(26.3 \%)$ & $2(12.5 \%)$ & \\
\hline$>20$ & $2(5.3 \%)$ & $1(6.3 \%)$ & \\
\hline Gleason score (sum) & & & 0.685 \\
\hline 7 & $23(60.5 \%)$ & $12(75.0 \%)$ & \\
\hline 8 & $7(18.4 \%)$ & $3(18.8 \%)$ & \\
\hline$\underline{9}$ & $7(18.4 \%)$ & $1(6.3 \%)$ & \\
\hline 10 & $1(2.6 \%)$ & 0 & \\
\hline NCCN risk group & & & 0.274 \\
\hline Intermediate & $20(52.6 \%)$ & $11(68.8 \%)$ & \\
\hline High & $18(47.4 \%)$ & $5(31.3 \%)$ & \\
\hline ADT & & & 0.766 \\
\hline Yes & $17(44.7 \%)$ & $6(37.5 \%)$ & \\
\hline No & $21(55.3 \%)$ & $10(62.5 \%)$ & \\
\hline Median duration of ADT (months) (range) & $6(4-25)$ & $6(6-12)$ & \\
\hline Pelvic lymph node radiation therapy & & & 0.163 \\
\hline Yes & $6(15.8 \%)$ & 0 & \\
\hline No & $32(84.2 \%)$ & $16(100 \%)$ & \\
\hline
\end{tabular}

PSA - prostate specific antigen, NCCN - the National Comprehensive Cancer Network, ADT - androgen deprivation therapy

sexual dysfunction was significantly associated with time from intervention $(p=0.041)$, peaking at $>30$ months for both dose groups.

\section{Vitality/quality of life}

Vitality score was not significantly different between the two groups over time $(p=0.37)$, or at any time-point after intervention (Figure 2). Also, QoL scores were not significantly different between the dosing groups with time $(p=0.71)$ (Figure 2). QoL scores peaked at the 0-2 months' time point in the hypofractionation group, and at 3-6 months in the standard fractionation group. Vitality scores peaked at 3-6 months in both groups.

\section{Discussion}

The results in our study suggest hypofractionated EBRT is feasible in combination with an HDR brachytherapy boost. There was no increase in acute or late toxicity as measured by AUASS and EPIC-CP for a hypofractionated EBRT regimen combined with HDR brachytherapy boost, compared to standard fractionation EBRT plus HDR boost.
When treating intermediate and high-risk prostate cancer with RT, EBRT with dose escalation via brachytherapy has been found to improve biochemical progression-free survival compared to dose escalation via EBRT (DE-EBRT). In the ACSCENDE-RT trial by Morris et al., all patients were randomized to receive $46 \mathrm{~Gy}$ to the pelvis with a conformal EBRT boost to a total of 78 Gy or the same pelvic dose of 46 Gy plus an LDR brachytherapy boost. The 9-year biochemical progression-free survival (b-PFS) in the DE-EBRT group was $62 \%$, compared to $83 \%$ for the EBRT plus LDR group. This improvement in b-PFS was at the cost of higher late grade 3 genitourinary toxicity in the LDR boost group. Additional, a multi-institutional retrospective cohort study of 1,809 men with localized Gleason 9 and 10 prostate cancer compared clinical outcomes in men treated with EBRT plus a brachytherapy boost and ADT vs. EBRT plus ADT vs. radical prostatectomy [19]. Five-year prostate cancer-specific mortality was significantly lower in the EBRT plus brachytherapy boost and ADT group. Furthermore, this group was found to have a longer time to distant metastasis compared to the EBRT and ADT and radical prostatectomy groups. Moreover, acceptable toxicity rates have been shown using this 
A

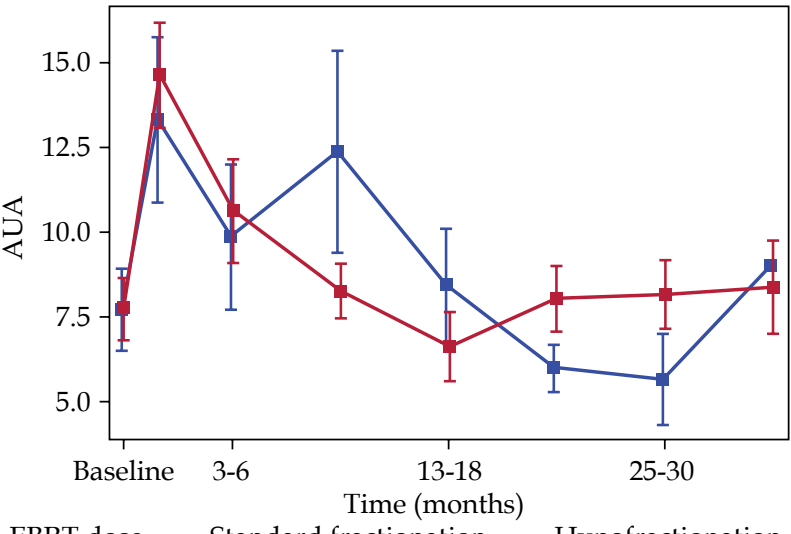

EBRT dose $\backsim$ Standard fractionation $\backsim$ Hypofractionation
B

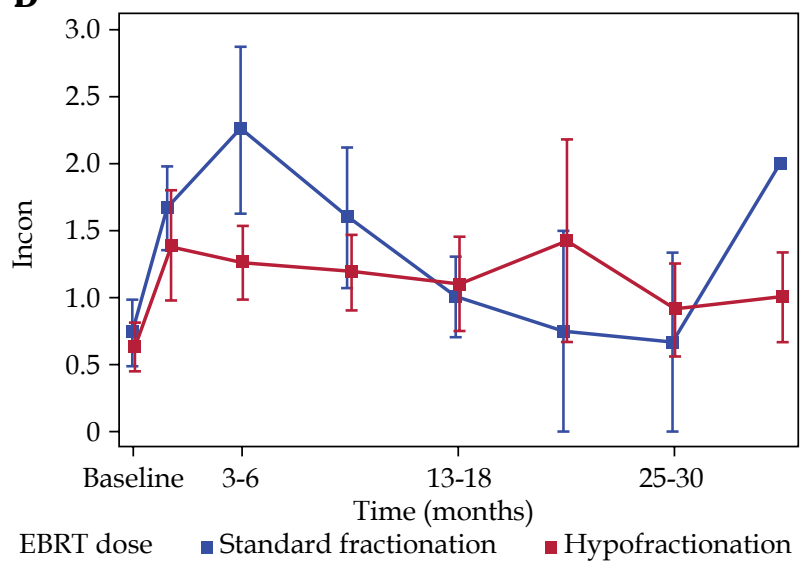

C

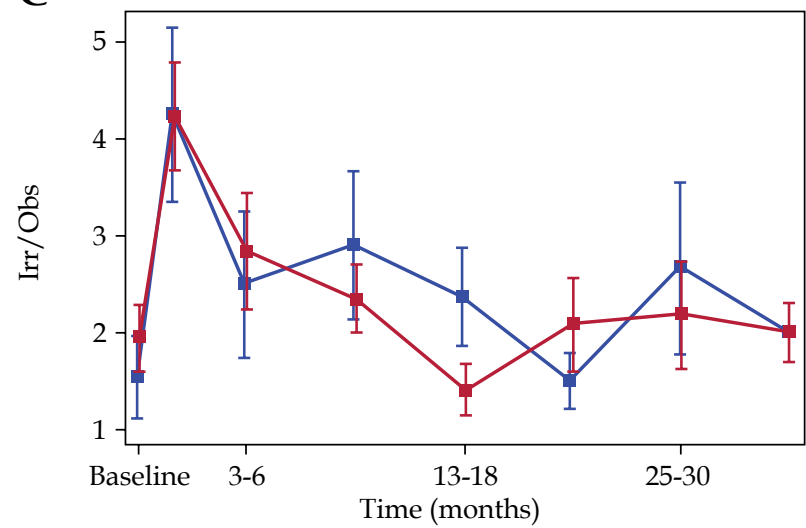

EBRT dose $\quad$ Standard fractionation Hypofractionation

Patient survey completion rates

\begin{tabular}{|c|c|c|c|c|c|c|c|c|}
\hline $\begin{array}{l}\text { Number of patients } \\
\text { who completed the survey }\end{array}$ & Baseline & $\begin{array}{c}0-2 \\
\text { months }\end{array}$ & $\begin{array}{c}3-6 \\
\text { months }\end{array}$ & $\begin{array}{c}\text { 7-12 } \\
\text { months }\end{array}$ & $\begin{array}{c}13-18 \\
\text { months }\end{array}$ & $\begin{array}{l}19-24 \\
\text { months }\end{array}$ & $\begin{array}{l}25-30 \\
\text { months }\end{array}$ & $\begin{array}{l}>30 \\
\text { months }\end{array}$ \\
\hline Standard fractionation & 38 & 26 & 20 & 26 & 19 & 16 & 11 & 16 \\
\hline Hypofractionation & 16 & 12 & 8 & 10 & 10 & 5 & 4 & 4 \\
\hline
\end{tabular}

Fig. 1. Average urinary function symptom scores over time by group A) American Urological Association Symptom Score

(AUASS), B) urinary incontinence (incon) score, C) irritability and obstructive symptoms (irr/obs) score

approach [20,21]. These results have led to a push for combination treatment in those with NCCN high-risk prostate cancer, along with select intermediate risk patients. An additional benefit of a brachytherapy boost is shortened overall treatment time for patients.

With a need for cost-effective, convenient, and effective treatments, hypofrationated EBRT is becoming increasingly utilized. Hypofractionated EBRT has been shown to be safe and efficacious when applied as monotherapy, with multiple randomized studies addressing this question. While RTOG 0415 included only patients with low-risk disease, PROFIT consisted of patients with intermediate-risk disease, and CHHiP and HYPRO included both intermediate- and high-risk patients $[13,16]$. In RTOG 0415, late grade 2 and 3 GI and GU toxicity was significantly higher in the hypofractionated group [16]. In HYPRO, cumulative grade 3 or worse late GU toxicity was significantly higher in the hypofractionation group than in the standard fractionation group. However, there was no significant difference in cumulative grade 3 or higher GI toxicity between the 2 groups [13]. However, in CHHiP, there was not a significant difference in grade 2 or higher GI or GU toxicity in the hypofractionation group and in PROFIT, there was no significant difference in grade 3 or higher GI or GU toxicity $[14,15]$. Together, these studies have proven the safety and efficacy of hypofractionated EBRT as prostate cancer monotherapy.

While less robust data exists for hypofractionated EBRT plus brachytherapy boost, an early report by Sathya et al. compared EBRT alone to EBRT plus a brachytherapy boost. In the EBRT alone group, a dose of $66 \mathrm{~Gy}$ in 33 fractions was delivered; in the combination group, patients received an EBRT dose of $40 \mathrm{~Gy}$ in 20 fractions plus an iridium implant delivering 35 Gy over 48 hours to the prostate. This study did report similar rates of acute and late toxicity between the groups [9]. Similar to our 
A

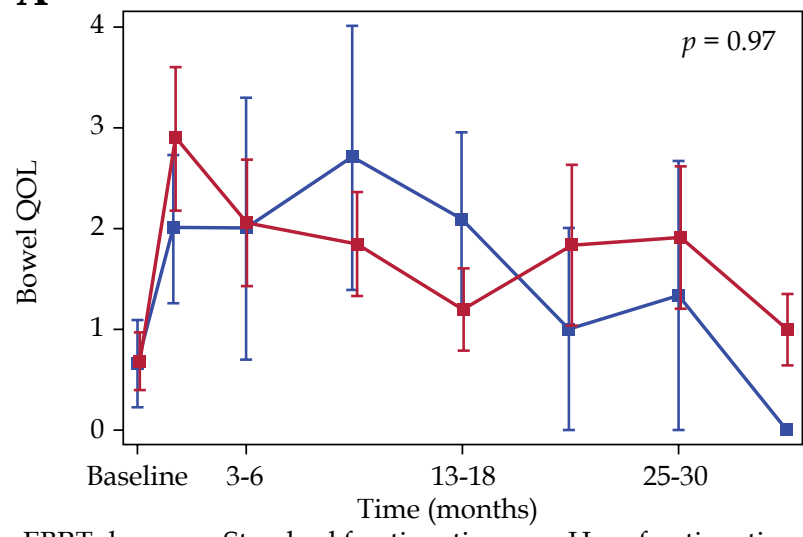

EBRT dose - Standard fractionation $\quad$ Hypofractionation

C

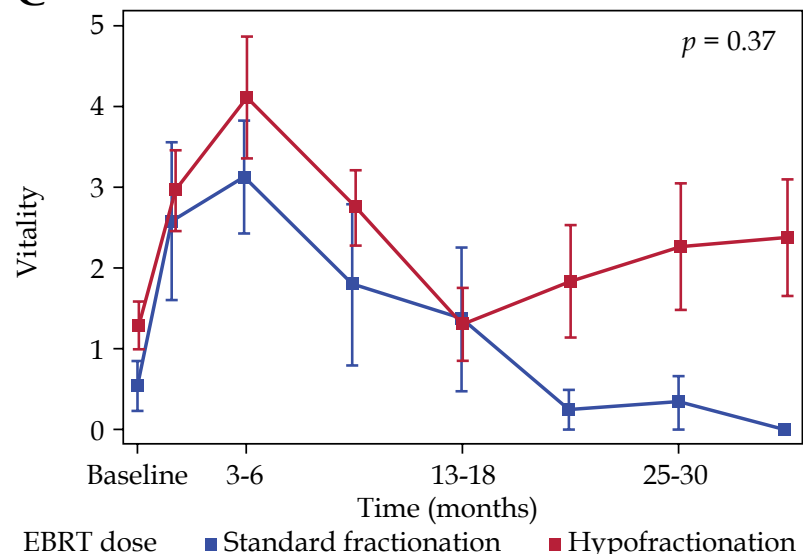

B

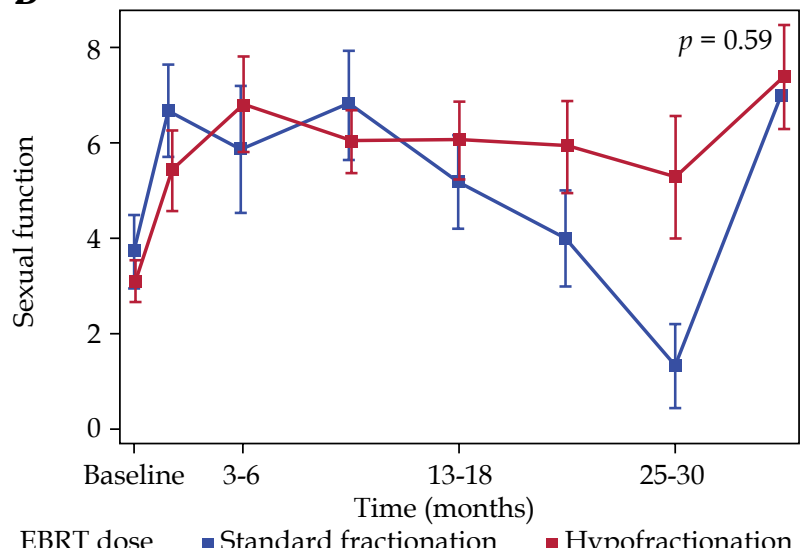

D

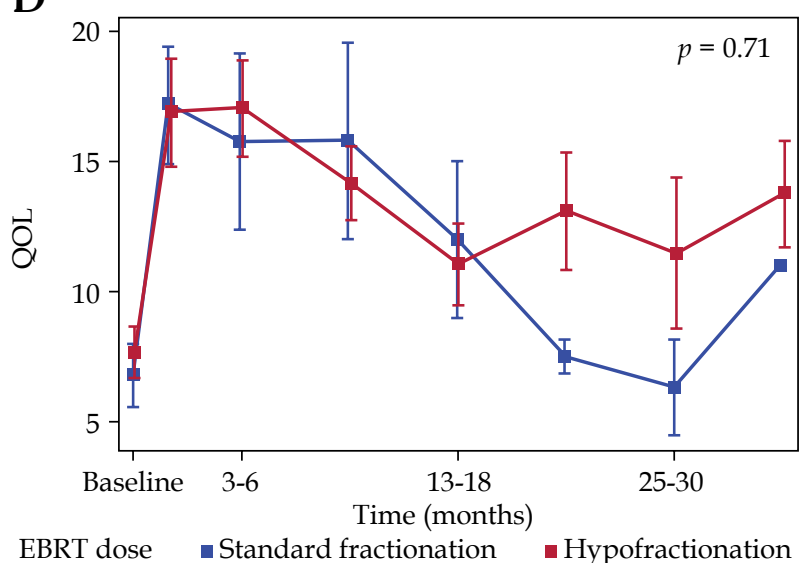

Fig. 2. Average bowel, sexual function, vitality, and quality of life (QoL) symptom scores over time by group: A) bowel QoL score, B) sexual function score, C) vitality score, D) overall Expanded Prostate Index for Prostate Cancer - Clinical Practice (EPIC-CP) QoL score

study, a more modern series by Morton et al. included patients receiving EBRT to $37.5 \mathrm{~Gy}$ in 15 fractions plus HDR boost to 15 Gy in 1 fraction [17]. This group was compared to patients receiving 45 Gy in 25 fractions with an HDR boost of $10 \mathrm{~Gy}$ in 2 fractions. While late urinary and bowel toxicity was similar between the two groups, the differing HDR dosing makes it difficult to compare the two EBRT dose groups in terms of toxicity. Morton et al. and Shahid et al. reported on the acute and long-term toxicity and health-related QoL of intermediate-risk prostate cancer patients who received an HDR boost of $15 \mathrm{~Gy}$ in 1 fraction, followed by hypofractionated EBRT to $37.5 \mathrm{~Gy}$ in 15 fractions in a single-institution phase II trial as mentioned above [22,23]. Both the IPSS and EPIC questionnaires were used for assessment. In this study, IPSS scores rose by 7 points acutely before improving, which mirrors what was seen in our study for both groups. Also, similar to our study, the greatest decline was seen in the EPIC sexual subdomain score, which continued to decline over time. The EPIC urinary, bowel, and vitality subdomain scores showed worsened function acutely before improving, with small absolute changes. The authors noted a majority of the decline occurred over the first 12 months. Again, this is comparable to our own study, in which function worsened acutely with small absolute changes in subdomain scores before improving. It is also important to note that changes in the incon subdomain score reflect urge incontinence rather than the stress incontinence commonly seen after prostatectomy. When there was a difference, the hypofractionation group generally experienced an earlier peak in subdomain scores in our study. We expect this is due to the slightly higher BED in this group. With the exception of sexual function, all EPIC subdomain scores showed absolute changes of $<3$ points over time for both groups in our cohort.

Though limited by its retrospective nature and small sample size, our study does offer a direct comparison of two fractionation schedules of EBRT in combination with an HDR brachytherapy boost in terms of patient-reported toxicity outcomes. This adds to the limited toxicity data regarding the use of a hypofractionated EBRT regimen in this field. Brachytherapy boost doses were not uniform in our study. The doses used represent the evolution of our practice to a higher dose per fraction with the 2-fraction regimen, and ultimately to a single fraction regimen for patient convenience. However, multiple studies have compared HDR fractionation schedules similar to our own showing no clinically significant differences in toxicity between varying regimens $[17,24,25,26]$. Furthermore, the cumulative BEDs used in our cohort have been associated with excellent oncologic outcomes [27]. 
In conclusion, our study suggests a hypofractionated regimen of EBRT can be delivered in combination with an HDR boost for patients with intermediate- and highrisk adenocarcinoma of prostate without increasing toxicity compared to standard fractionation EBRT with an HDR boost. This further supports current ACR guidelines, and has important implications in terms of patient convenience and resource utilization. A clinical trial comparing these two fractionation schemes with a brachytherapy boost is warranted to confirm these results.

\section{Disclosure}

The authors report no conflict of interest.

\section{References}

1. Kuban DA, Tucker SL, Dong L et al. Long-term results of the M. D. Anderson randomized dose-escalation trial for prostate cancer. Int J Radiat Oncol Biol Phys 2008; 70: 67-74.

2. Zietman AL, Bae K, Slater JD et al. Randomized trial comparing conventional-dose with high-dose conformal radiation therapy in early-stage adenocarcinoma of the prostate: longterm results from proton radiation oncology group/american college of radiology 95-09. J Clin Oncol 2010; 28: 1106-1111.

3. Dearnaley DP, Sydes MR, Graham JD et al. Escalated-dose versus standard-dose conformal radiotherapy in prostate cancer: first results from the MRC RT01 randomised controlled trial. Lancet Oncol 2007; 8: 475-487.

4. Peeters ST, Heemsbergen WD, Koper PC et al. Dose-response in radiotherapy for localized prostate cancer: results of the Dutch multicenter randomized phase III trial comparing $68 \mathrm{~Gy}$ of radiotherapy with 78 Gy. J Clin Oncol 2006; 24: 1990-1996.

5. Beckendorf V, Guerif S, Le Prise E et al. 70 Gy versus 80 Gy in localized prostate cancer: 5-year results of GETUG 06 randomized trial. Int J Radiat Oncol Biol Phys 2011; 80: 1056-1063.

6. Zelefsky MJ, Chan H, Hunt M et al. Long-term outcome of high dose intensity modulated radiation therapy for patients with clinically localized prostate cancer. J Urol 2006; 176: 1415-1419.

7. Zelefsky MJ, Kollmeier M, Cox B et al. Improved clinical outcomes with high-dose image guided radiotherapy compared with non-IGRT for the treatment of clinically localized prostate cancer. Int J Radiat Oncol Biol Phys 2012; 84: 125-129.

8. Spratt DE, Pei X, Yamada J et al. Long-term survival and toxicity in patients treated with high-dose intensity modulated radiation therapy for localized prostate cancer. Int J Radiat Oncol Biol Phys 2013; 85: 686-692.

9. Sathya JR, Davis IR, Julian JA et al. Randomized trial comparing iridium implant plus external-beam radiation therapy with external-beam radiation therapy alone in node-negative locally advanced cancer of the prostate. J Clin Oncol 2005; 23: 1192-1199.

10. Hoskin PJ, Rojas AM, Bownes PJ et al. Randomised trial of external beam radiotherapy alone or combined with highdose-rate brachytherapy boost for localised prostate cancer. Radiother Oncol 2012; 103: 217-222.

11. Morris WJ, Tyldesley S, Rodda S et al. Androgen Suppression Combined with Elective Nodal and Dose Escalated Radiation Therapy (the ASCENDE-RT Trial): An Analysis of Survival Endpoints for a Randomized Trial Comparing a Low-Dose-Rate Brachytherapy Boost to a Dose-Escalated External Beam Boost for High- and Intermediate-risk Prostate Cancer. Int J Radiat Oncol Biol Phys 2017; 98: 275-285.

12. Brenner DJ, Hall EJ. Fractionation and protraction for radiotherapy of prostate carcinoma. Int J Radiat Oncol Biol Phys 1999; 43: 1095-1101.
13. Incrocci L, Wortel RC, Alemayehu WG et al. Hypofractionated versus conventionally fractionated radiotherapy for patients with localised prostate cancer (HYPRO): final efficacy results from a randomised, multicentre, open-label, phase 3 trial. Lancet Oncol 2016; 17: 1061-1069.

14. Catton CN, Lukka H, Gu CS et al. Randomized Trial of a Hypofractionated Radiation Regimen for the Treatment of Localized Prostate Cancer. J Clin Oncol 2017; 35: 1884-1890.

15. Dearnaley D, Syndikus I, Mossop H et al. Conventional versus hypofractionated high-dose intensity-modulated radiotherapy for prostate cancer: 5-year outcomes of the randomised, non-inferiority, phase $3 \mathrm{CHHiP}$ trial. Lancet Oncol 2016; 17: 1047-1060.

16. Lee WR, Dignam JJ, Amin MB et al. Randomized Phase III Noninferiority Study Comparing Two Radiotherapy Fractionation Schedules in Patients With Low-Risk Prostate Cancer. J Clin Oncol 2016; 34: 2325-2332.

17. Morton G, Loblaw A, Cheung P et al. Is single fraction $15 \mathrm{~Gy}$ the preferred high dose-rate brachytherapy boost dose for prostate cancer? Radiother Oncol 2011; 100: 463-467.

18. Hsu IC, Yamada Y, Assimos DG et al. ACR Appropriateness Criteria high-dose-rate brachytherapy for prostate cancer. Brachytherapy 2014; 13: 27-31.

19. Kishan AU, Cook RR, Ciezki JP et al. Radical Prostatectomy, External Beam Radiotherapy, or External Beam Radiotherapy With Brachytherapy Boost and Disease Progression and Mortality in Patients With Gleason Score 9-10 Prostate Cancer. JAMA 2018; 319: 896-905.

20. Halkett GKB, Short M, Aoun $S$ et al. What pelvic radiation disease symptoms are experienced by patients receiving external beam radiotherapy and a high-dose-rate brachytherapy boost for prostate cancer? J Contemp Brachytherapy 2017; 9: 393-402.

21. Rodda S, Tyldesley S, Morris WJ et al. ASCENDE-RT: An Analysis of Treatment-Related Morbidity for a Randomized Trial Comparing a Low-Dose-Rate Brachytherapy Boost with a DoseEscalated External Beam Boost for High- and IntermediateRisk Prostate Cancer. Int J Radiat Oncol Biol Phys 2017; 98: 286-295.

22. Shahid N, Loblaw A, Chung HT et al. Long-term Toxicity and Health-related Quality of Life after Single-fraction High Dose Rate Brachytherapy Boost and Hypofractionated External Beam Radiotherapy for Intermediate-risk Prostate Cancer. Clin Oncol (R Coll Radiol) 2017; 29: 412-420.

23. Morton GC, Loblaw DA, Chung $\mathrm{H}$ et al. Health-related quality of life after single-fraction high-dose-rate brachytherapy and hypofractionated external beam radiotherapy for prostate cancer. Int J Radiat Oncol Biol Phys 2011; 80: 1299-1305.

24. Helou J, D'Alimonte L, Loblaw A et al. High dose-rate brachytherapy boost for intermediate risk prostate cancer: Long-term outcomes of two different treatment schedules and early biochemical predictors of success. Radiother Oncol 2015; 115: 84-89.

25. Falk AT, Demontoy S, Chamorey E et al. High-dose-rate brachytherapy boost for prostate cancer: Comparison of three different fractionation schemes. Brachytherapy 2017; 16: 993-999.

26. Vigneault E, Mbodji $K$, Magnan S et al. High-dose-rate brachytherapy boost for prostate cancer treatment: Different combinations of hypofractionated regimens and clinical outcomes. Radiother Oncol 2017; 124: 49-55.

27. Okamoto K, Wada A, Kohno N. High biologically effective dose radiation therapy using brachytherapy in combination with external beam radiotherapy for high-risk prostate cancer. J Contemp Brachytherapy 2017; 9: 1-6. 